\title{
Association of Preoperative Prognostic Nutritional Index and Postoperative Acute Kidney Injury in Patients Who Underwent Hepatectomy for Hepatocellular Carcinoma
}

\author{
Ji Hoon Sim, In-Gu Jun, Young-Jin Moon $\mathbb{1}$, A Rom Jeon, Sung-Hoon Kim, Bomi Kim and Jun-Gol Song *(D)
}

check for updates

Citation: Sim, J.H.; Jun, I.-G.; Moon, Y.-J.; Jeon, A.R.; Kim, S.-H.; Kim, B.;

Song, J.-G. Association of

Preoperative Prognostic Nutritional

Index and Postoperative Acute

Kidney Injury in Patients Who

Underwent Hepatectomy for

Hepatocellular Carcinoma. J. Pers.

Med. 2021, 11, 428. https://

doi.org/10.3390/jpm11050428

Academic Editor: Susan Sumner

Received: 23 April 2021

Accepted: 17 May 2021

Published: 18 May 2021

Publisher's Note: MDPI stays neutral with regard to jurisdictional claims in published maps and institutional affiliations.

Copyright: (C) 2021 by the authors Licensee MDPI, Basel, Switzerland. This article is an open access article distributed under the terms and conditions of the Creative Commons Attribution (CC BY) license (https:/ / creativecommons.org/licenses/by/ $4.0 /)$.
Department of Anesthesiology and Pain Medicine, Asan Medical Center, University of Ulsan College of Medicine, Seoul 05505, Korea; atlassjh@hanmail.net (J.H.S.); igjun@amc.seoul.kr (I.-G.J.); yjmoon@amc.seoul.kr (Y.-J.M.); atomy0429@gmail.com (A.R.J.); shkimans@gmail.com (S.-H.K.); dudnd5@gmail.com (B.K.)

* Correspondence: jungol.song@amc.seoul.kr; Tel.: +82-2-3010-3869

\begin{abstract}
Various biological indicators are reportedly associated with postoperative acute kidney injury (AKI) in the surgical treatment of hepatocellular carcinoma (HCC). However, only a few studies have evaluated the association between the preoperative prognostic nutritional index (PNI) and postoperative AKI. This study evaluated the association of the preoperative PNI and postoperative AKI in HCC patients. We retrospectively analyzed 817 patients who underwent open hepatectomy between December 2007 and December 2015. Multivariate regression analysis was performed to evaluate the association between the PNI and postoperative AKI. Additionally, we evaluated the association between the PNI and outcomes such as postoperative renal replacement therapy (RRT) and mortality. Cox regression analysis was performed to assess the risk factors for one-year and five-year mortality. In the multivariate analysis, high preoperative PNI was significantly associated with a lower incidence of postoperative AKI (odds ratio (OR): 0.92, 95\% confidence interval (CI): 0.85 to $0.99, p=0.021$ ). Additionally, diabetes mellitus and the use of synthetic colloids were significantly associated with postoperative AKI. PNI was associated with postoperative RRT (OR: 0.76, 95\% CI: 0.60 to $0.98, p=0.032$ ) even after adjusting for other potential confounding variables. In the Cox regression analysis, high PNI was significantly associated with low one-year mortality (Hazard ratio (HR): 0.87, 95\% CI: 0.81 to $0.94, p<0.001$ ), and five-year mortality (HR: 0.93, 95\% CI: 0.90-0.97, $p<0.001$ ). High preoperative PNI was significantly associated with a lower incidence of postoperative AKI and low mortality. These results suggest that the preoperative PNI might be a predictor of postoperative AKI and surgical prognosis in HCC patients undergoing open hepatectomy.
\end{abstract}

Keywords: acute kidney injury; hepatocellular carcinoma; prognostic nutritional index; mortality

\section{Introduction}

Hepatectomy is a major intra-abdominal general surgery with a high risk of postoperative AKI, which is unique due to the patient's underlying disease, as well as surgical and anesthetic management considerations [1]. The reported incidence of AKI in patients who underwent hepatic resection for HCC is $15 \%$ to $20 \%$ [2-4]. AKI increases morbidity, mortality, and hospital costs [4-6]. AKI is also associated with a risk of developing chronic kidney disease (CKD) [7]. Therefore, there have been many studies to identify the risk factors for postoperative AKI in HCC patients [8-13]. However, until now, there have been few well-established risk factors for postoperative AKI in the context of hepatectomy.

The prognostic nutritional index (PNI), a biological marker, which can be determined through the serum albumin level and total lymphocyte count in peripheral blood, reflects a patient's nutritional and inflammatory status [14]. The PNI was reportedly associated with survival or complications in various diseases, including cancer [15-18]. Recent studies have also indicated that the PNI is an effective predictor of prognosis in patients with HCC after hepatectomy $[19,20]$. 
However, only a few studies have reported the association between the preoperative PNI and postoperative AKI in HCC patients. Therefore, we aimed to evaluate the prognostic role of the preoperative PNI in developing postoperative AKI in HCC patients who had undergone hepatectomy. We also assessed surgical outcomes such as the length of hospital stay, postoperative intensive care unit (ICU) admission, and mortality rate.

\section{Materials and Methods}

\subsection{Study Design \& Patient Population}

We retrospectively reviewed 817 patients diagnosed with HCC according to the 10th revision of the International Classification of Diseases (ICD-10) guidelines, who underwent liver resection between December 2007 and December 2015. The institutional review board of Asan Medical Center (protocol number: 2021-0243) approved this study and waived the need for written consent. Adult patients aged over 18 years who underwent open hepatectomy were included in the study. All surgeries were planned and performed by a single surgeon. The exclusion criteria were as follows: patients aged $<18$ or $\geq 80$ years, patients with severe cardiopulmonary or chronic kidney disease, patients who had already received renal replacement therapy (RRT), patients who required intervention in the urinary system during surgery, patients who underwent emergency surgery, and patients with incomplete data or missing PNI or serum creatinine values.

\subsection{Anesthetic \& Surgical Technique}

For the induction of general anesthesia, we administered an intravenous bolus injection of thiopental sodium (4 to $5 \mathrm{mg} / \mathrm{kg}$ ) or propofol (1 to $2 \mathrm{mg} / \mathrm{kg}$ ). After loss of consciousness, rocuronium ( 0.6 to $1.2 \mathrm{mg} / \mathrm{kg}$ ) was administered for muscle relaxation. Before tracheal intubation, 1 to $2 \mu \mathrm{g} / \mathrm{kg}$ of fentanyl was administered as an intravenous bolus injection, and maintenance of anesthesia was achieved with 2 to $4 \mathrm{vol} \%$ sevoflurane in $50 \%$ air/oxygen. After insertion of the arterial and central venous lines, invasive monitoring of arterial and central venous pressure was routinely performed. The patient was mechanically ventilated with a tidal volume of 6 to $8 \mathrm{~mL} / \mathrm{kg}$, and the end-expiratory carbon dioxide partial pressure was adjusted to maintain a value of 35 to $40 \mathrm{mmHg}$. During anesthesia, crystalline solutions (Ringer's lactate solution or plasma solution) or colloidal solutions (5\% albumin or synthetic colloids (Voluven ${ }^{\circledR}$; Fresenius Kabi, Bad Homburg, Germany)) were administered, and the total volume of synthetic colloids did not exceed $20 \mathrm{~mL} / \mathrm{kg}$. When the plasma hemoglobin $(\mathrm{Hb})$ level was less than $8 \mathrm{~g} / \mathrm{dL}$ during surgery, packed red blood cell (RBC) transfusion was performed. The $\mathrm{Hb}$ level in patients with ischemic heart disease was maintained at $>10 \mathrm{~g} / \mathrm{dL}$. Vasopressors such as ephedrine or phenylephrine were administered when the mean arterial blood pressure was less than $65 \mathrm{mmHg}$, and inotropic agents such as norepinephrine were administered when the vasopressor was ineffective, under the clinical judgment of an anesthesiologist.

Open abdominal surgery was performed through a J-shaped incision. Liver parenchymal transection was performed using an ultrasonic surgical aspirator (CUSA ${ }^{\circledR}$ Excel; Valleylab Inc., Boulder, CO, USA). After parenchymal dissection, the hepatic vein was cut and ligated with a stapler or clamp and then sutured. Liver resection was performed based on the definition by Couinaud classification [21]. Minor resection was defined when hepatic resection was limited to two or fewer segments, and the others were defined as a major resection [22]. Right anterior and posterior sectionectomy were classified as major resection as advanced techniques and a longer operation time are frequently required for those types of hepatic resections [23]. Hemostasis was achieved using electrocoagulation, clips, argon beam, or nonabsorbable sutures. Systematic placement of abdominal drainage was routinely performed during surgery.

After surgery, crystalloids and colloids were administered appropriately to maintain fluid balance and normal kidney function. Management with sodium restriction and judicious use of diuretic therapy were carried out when there was new onset postoperative ascites, and we did not use diuretics routinely. Blood tests were performed daily 
for three days after surgery to measure the patient's complete blood count, biochemical and electrolyte levels, and inflammation levels. We routinely used postoperative intravenous patient-controlled analgesia (PCA) for pain control. In some cases, hepatoprotective agents such as branched-chain amino acids (BCAA) were administered to support protein synthesis and regeneration of the remnant liver.

\subsection{Clinical Data Collection and Outcome Assessments}

Demographic data and data on perioperative variables were collected from the electronic medical record system. Demographic data included age, sex, weight, body mass index, TNM staging, number of tumors, size of tumors, lymph node invasion, and distant metastasis. Data regarding the presence of comorbid diseases, such as diabetes mellitus (DM), hypertension, coronary artery disease, and cerebrovascular accident, liver cirrhosis, indocyanine green retention rate at $15 \mathrm{~min}$ (ICG R15), and the model for end-stage liver disease (MELD) and Child-Turcotte-Pugh scores, which indicate the severity of liver disease, were also collected.

Laboratory values included preoperative white blood cell count, $\mathrm{Hb}$, platelet count, prothrombin time, albumin, serum creatinine (sCr), estimated glomerular filter rate (eGFR), total bilirubin, aspartate aminotransferase (AST), alanine aminotransferase (ALT), and sodium. Serum creatinine levels were checked daily, from postoperative day 1 to day 7 , to confirm AKI. Preoperative PNI values were also collected. The PNI was calculated using the following formula: $[10 \times$ serum albumin $(\mathrm{g} / \mathrm{dL})]+[0.005 \times$ total lymphocyte count $\left(\right.$ per $\left.\left.\mathrm{mm}^{3}\right)\right]$. The total blood counts of all patients were determined preoperatively, less than two days after admission, and prior to surgery. Intraoperative variables included operation time, total fluids, administered crystalloids and colloids, RBC transfusion, diuretics, and urine output. Data on postoperative AKI incidence and grade, postoperative RRT, posthepatectomy liver failure (PHLF), hospital stay, ICU admission, prolonged ICU stay ( $\geq$ two days), and one-year mortality (calculated from the date of surgery to one-year follow-up), and overall mortality (determined from the date of surgery to the last follow-up) records were also collected.

\subsection{Primary and Secondary Outcomes}

The primary outcome was the association between postoperative AKI and the preoperative PNI. The secondary outcome was the association between the preoperative PNI and outcomes, such as postoperative RRT, PHLF, ICU admission, one-year, five-year, and overall mortality. Additionally, we evaluated the risk factors associated with postoperative AKI and one-year, and five-year mortality. The Kidney Disease Improving Global Outcomes classification defines $\mathrm{AKI}$ as an increase in $\mathrm{sCr} \geq 1.5$ times the baseline value, within seven days prior to surgery or increase in $\mathrm{sCr}$ by $\geq 0.3 \mathrm{mg} / \mathrm{dL}$ within $48 \mathrm{~h}$ [24]. PHLF was defined by the International Study Group of Liver Surgery (ISGLS) [25].

\subsection{Statistical Analysis}

Categorical data were analyzed using the chi-squared test or Fisher's exact test, and continuous data were evaluated using the independent $t$-test or Mann-Whitney $U$ test. The data are appropriately presented as mean \pm standard deviation (SD), median of the quartile range, or numbers with proportions. We used the multivariate logistic regression analysis to determine the association between the preoperative PNI and AKI. In univariate analysis, all variables with $p$-values less than 0.1 were included in the multivariate analysis. Cox regression analysis was used to evaluate the adjusted risk ratio of one-year and five-year mortality. The proportional hazard assumption was tested using Schoenfeld's residual test. We set the PNI cutoff value for mortality rate to 45 , guided by receiver operating characteristic analysis (AUC: 0.731 , sensitivity: 74.58, specificity: 61.21). The Kaplan-Meier method was used to analyze the cumulative one-year and five-year survival between the PNI $<45$ and PNI $\geq 45$ groups. The log-rank test was used to evaluate the change between curves. All $p$-values less than 0.05 were considered statistically significant. Data 
manipulation and statistical analyses were performed using the IBM SPSS Statistics for Windows, version 22.0 (IBM Corporation, Armonk, NY, USA).

\section{Results}

Of the 921 enrolled patients, 104 were excluded based on the study criteria. Finally, a total of 817 patients were enrolled in this study (Figure 1).

Open hepatocellular cancer surgery patients between December 1, 2007 and December 28, 2015 ( $n=921$ )

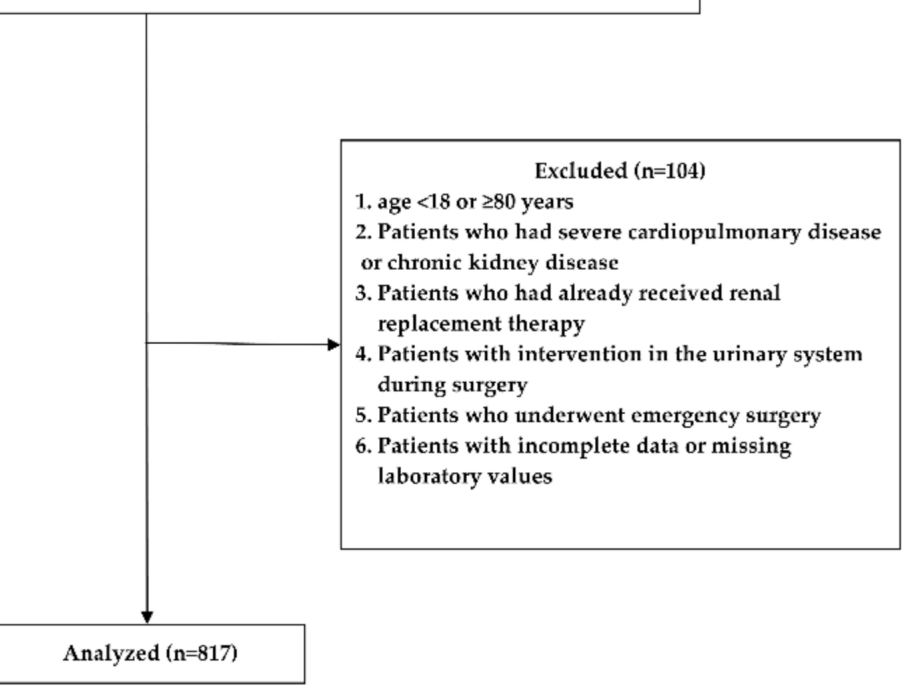

Figure 1. Study flow chart.

Table 1 shows the demographic data, perioperative variables, and surgical outcomes of the study population. The postoperative AKI incidence rate was $7.2 \%(59 / 817)$; most of the cases were grade 1 , and grades 2 and 3 occurred in four and three patients, respectively. The percentage of patients who underwent postoperative RRT was $1.0 \%(8 / 817)$, and the incidence rate of PHLF was $10.3 \%(84 / 817)$. The average length of hospital stay was 20.69 days, the ICU admission rate was $7.5 \%(61 / 817)$, and the one-year and overall mortality were $7.2 \%(59 / 817)$ and $33.3 \%(272 / 817)$, respectively (Table 1$)$. The median follow-up durations for determining the overall mortality and postoperative time after RRT were 3.41 (1.84 to 5.16 ) years and 7.00 (4.50 to 12.75$)$ days, respectively.

Table 1. Demographic and perioperative variables of the study population.

\begin{tabular}{cc}
\hline & Study Population $(\boldsymbol{n}=\mathbf{8 1 7})$ \\
\hline Preoperative variables & \\
Age; year & $62.60 \pm 11.83$ \\
Sex; male & $512(57.9)$ \\
Weight; $\mathrm{kg}$ & $59.56 \pm 9.84$ \\
BMI; kg m $^{-2}$ & $22.90 \pm 3.17$ \\
TNM staging & \\
1 & $489(59.9)$ \\
2 & $48(5.9)$ \\
3A & $16(2.0)$ \\
3B & $5(0.6)$ \\
$4 \mathrm{~A}$ & $233(28.5)$ \\
4B & $26(3.2)$ \\
Solitary & $715(87.5)$ \\
$\geq 2$ & $102(12.5)$ \\
\hline
\end{tabular}


Table 1. Cont.

\begin{tabular}{|c|c|}
\hline & Study Population $(n=817)$ \\
\hline Tumor size; cm & $4.99 \pm 4.02$ \\
\hline \multicolumn{2}{|l|}{ Lymph node invasion } \\
\hline 0 & $567(69.4)$ \\
\hline$\geq 1$ & $250(30.6)$ \\
\hline Metastasis & $29(3.5)$ \\
\hline DM & $50(6.1)$ \\
\hline HTN & $56(6.9)$ \\
\hline CAD & $6(0.7)$ \\
\hline CVA & $2(0.2)$ \\
\hline Liver cirrhosis & $298(36.5)$ \\
\hline \multicolumn{2}{|l|}{ ICG R15 $(n=731)$ * } \\
\hline$<10$ & $233(31.9)$ \\
\hline $10-30$ & $476(65.1)$ \\
\hline$\geq 30$ & $20(2.7)$ \\
\hline MELD scores & $7.19 \pm 1.11$ \\
\hline CTP scores & $5.29 \pm 0.49$ \\
\hline \multicolumn{2}{|l|}{ Laboratory variables } \\
\hline White blood cell & $5.46 \pm 1.81$ \\
\hline Hemoglobin & $13.94 \pm 1.59$ \\
\hline Platelet & $166.81 \pm 70.17$ \\
\hline Prothrombin time & $1.04 \pm 0.08$ \\
\hline Albumin $\left(\mathrm{g} \cdot \mathrm{dL}^{-1}\right)$ & $3.78 \pm 0.40$ \\
\hline Creatinine $\left(\mathrm{mg} \cdot \mathrm{dL}^{-1}\right)$ & $0.82 \pm 0.16$ \\
\hline $\mathrm{eGFR}\left(\mathrm{mL} / \mathrm{min} / 1.73 \mathrm{~m}^{2}\right)$ & $78.88 \pm 8.86$ \\
\hline Total bilirubin & $0.80 \pm 0.38$ \\
\hline AST & $42.24 \pm 34.57$ \\
\hline ALT & $38.35 \pm 29.14$ \\
\hline Sodium & $139.72 \pm 2.56$ \\
\hline PNI & $46.76 \pm 5.64$ \\
\hline \multicolumn{2}{|l|}{ Intraoperative variables } \\
\hline Operation time (min) & $269.18 \pm 75.08$ \\
\hline \multicolumn{2}{|l|}{ Type of liver resection } \\
\hline Minor surgery & $515(63.0)$ \\
\hline Major surgery & $302(37.0)$ \\
\hline Total fluids (mL/kg) & $40.09 \pm 19.58$ \\
\hline Crystalloids (mL/kg) & $34.96 \pm 16.76$ \\
\hline Colloids (mL/kg) & $5.13 \pm 6.05$ \\
\hline Colloid use & $446(54.6)$ \\
\hline RBC transfusion & $64(7.8)$ \\
\hline Units of infused RBC & $0.26 \pm 1.29$ \\
\hline Urine output (mL/kg/h) & $1.77 \pm 1.16$ \\
\hline \multicolumn{2}{|l|}{ Surgical outcomes } \\
\hline Postoperative AKI & $59(7.2)$ \\
\hline grade 1 & $52(6.4)$ \\
\hline grade 2 & $4(0.5)$ \\
\hline grade 3 & $3(0.4)$ \\
\hline Postoperative RRT & $8(1.0)$ \\
\hline PHLF & $84(10.3)$ \\
\hline Hospital stays & $20.69 \pm 13.07$ \\
\hline ICU admission & $61(7.5)$ \\
\hline One-year mortality & $59(7.2)$ \\
\hline Five-year mortality & $209(25.6)$ \\
\hline Overall mortality & $272(33.3)$ \\
\hline
\end{tabular}

* The total number is 731 due to missing data. BMI: body mass index; DM: diabetes mellitus; HTN: hypertension; CAD: coronary artery disease; CVA: cerebrovascular accident; ICG R15, indocyanine green retention rate at $15 \mathrm{~min}$ MELD: model for end-stage liver disease; CTP: Child-Turcotte-Pugh; eGFR: estimated glomerular filtration rate; AST: aspartate aminotransferase; ALT: alanine aminotransferase; PNI: prognostic nutritional index; RBC: red blood cell; AKI: acute kidney injury; RRT: renal replacement therapy; PHLF: post-hepatectomy liver failure; ICU: intensive care unit. Values are expressed as mean \pm standard deviation, median (interquartile range), or $\mathrm{n}$ (proportion). 


\subsection{Primary Outcomes}

In the multivariate analysis, high preoperative PNI was significantly associated with a lower incidence of postoperative AKI (odds ratio (OR): 0.92, 95\% confidence interval (CI): 0.85 to $0.99, p=0.021$ ). Additionally, DM (OR: $2.77,95 \%$ CI: 1.16 to $6.58, p=0.022$ ) and the use of synthetic colloids (OR: $1.99,95 \%$ CI: 1.05 to $3.80, p=0.036)$ were significantly associated with a high incidence of postoperative AKI (Table 2).

Table 2. Univariate and multivariate logistic regression analyses of acute kidney injury.

\begin{tabular}{|c|c|c|c|c|c|c|}
\hline & \multicolumn{3}{|c|}{ Univariate } & \multicolumn{3}{|c|}{ Multivariate } \\
\hline & OR & $95 \% \mathrm{CI}$ & $p$-Value & OR & $95 \% \mathrm{CI}$ & $p$-Value \\
\hline PNI & 0.93 & $0.89-0.98$ & 0.004 & 0.92 & $0.85-0.99$ & 0.021 \\
\hline Age (years) & 1.02 & 0.99-1.04 & 0.261 & 1.01 & $0.98-1.04$ & 0.496 \\
\hline Sex (male) & 1.81 & $0.76-4.30$ & 0.178 & 2.06 & $0.81-5.26$ & 0.130 \\
\hline BMI & 1.07 & $0.98-1.17$ & 0.125 & 1.09 & $0.98-1.21$ & 0.098 \\
\hline DM & 2.67 & $1.19-6.00$ & 0.017 & 2.77 & $1.16-6.58$ & 0.022 \\
\hline HTN & 1.60 & $0.66-3.91$ & 0.300 & & & \\
\hline MELD scores & 1.19 & $0.96-1.47$ & 0.116 & 1.01 & $0.79-1.30$ & 0.925 \\
\hline CTP scores & 1.52 & $0.93-2.47$ & 0.090 & 0.92 & $0.46-1.83$ & 0.803 \\
\hline TNM staging & & & 0.470 & & & 0.653 \\
\hline 1 & $\begin{array}{c}1.00 \\
\text { (Ref.) }\end{array}$ & & & $\begin{array}{c}1.00 \\
\text { (Ref.) }\end{array}$ & & \\
\hline 2 & 1.71 & $0.68-4.28$ & 0.254 & 1.35 & $0.51-3.57$ & 0.548 \\
\hline 3 & 0.65 & $0.15-2.79$ & 0558 & 0.54 & $0.12-2.44$ & 0.425 \\
\hline $4 \mathrm{~A}$ & 0.67 & $0.34-1.30$ & 0.237 & 0.55 & $0.27-1.14$ & 0.111 \\
\hline $4 \mathrm{~B}$ & 1.09 & $0.25-4.80$ & 0.913 & 0.63 & $0.13-3.09$ & 0.570 \\
\hline Operation time (min) & 1.01 & $1.00-1.01$ & $<001$ & 1.00 & $1.00-1.01$ & 0.061 \\
\hline Type of liver resection & & & 0.957 & & & \\
\hline Minor surgery & $\begin{array}{c}1.00 \\
\text { (Ref.) }\end{array}$ & & & & & \\
\hline Major surgery & 1.02 & $0.59-1.76$ & & & & \\
\hline Total fluids (mL/kg) & 1.02 & $1.00-1.03$ & 0.005 & 1.00 & $0.98-1.02$ & 0.778 \\
\hline Synthetic colloid use & 2.16 & $1.21-3.87$ & 0.009 & 1.99 & $1.05-3.80$ & 0.036 \\
\hline Urine output (mL/kg/h) & 0.82 & $0.63-1.07$ & 0.152 & & & \\
\hline $\mathrm{RBC}$ transfusion & 1.20 & $1.06-1.36$ & 0.005 & 1.18 & $0.45-3.08$ & 0.737 \\
\hline Albumin $\left(\mathrm{g} \cdot \mathrm{dL}^{-1}\right)$ & 0.33 & $0.17-0.62$ & $<001$ & & & \\
\hline
\end{tabular}

OR: odds ratio; CI: confidence interval; PNI: prognostic nutritional index; BMI: body mass index; DM: diabetes mellitus; HTN: hypertension; MELD: model for end-stage liver disease; CTP: Child-Turcotte-Pugh; RBC: red blood cell. Values are expressed as mean \pm standard deviation, median (interquartile range), or $\mathrm{n}$ (proportion).

\subsection{Secondary Outcomes}

In the Cox regression analysis of one-year mortality, high preoperative PNI was significantly associated with low one-year mortality (HR: $0.87,95 \%$ CI: 0.81 to $0.94, p<0.001$; Table 3). Moreover, TNM stages 3, 4A, and 4B (HR: 3.67, 95\% CI: 1.14 to 11.81, $p=0.030$ in stage 3; HR: $8.85,95 \%$ CI: 4.29 to $18.22, p<0.001$ in stage 4 A; HR: $9.26,95 \%$ CI: 3.42 to 25.11 , $p<0.001$ in stage $4 \mathrm{~B}$ ) were significantly associated with high one-year mortality (Table 3 ).

In the Cox regression analysis of five-year mortality, high preoperative PNI was significantly associated with low five-year mortality (HR: 0.93, 95\% CI: 0.90-0.97, $p<0.001$; Table 4). Moreover, MELD scores (HR: 1.16, 95\% CI: 1.03-1.30, $p=0.015$; Table 4), TNM stages 2, 4A, and 4B (HR: 1.91, 95\% CI: 1.07-3.40, $p=0.028$ in stage 2; HR: 3.73, 95\% CI: 2.72-5.11, $p<0.001$ in stage 4A; HR: 3.35, 95\% CI: 1.80-6.22, $p<0.001$ in stage 4B), and synthetic colloid use (HR: $1.48,95 \%$ CI: 1.08-2.03, $p=0.015$; Table 4) were significantly associated with a high five-year mortality (Table 4 ). 
Table 3. Cox regression analyses of one-year mortality.

\begin{tabular}{|c|c|c|c|c|c|c|}
\hline & \multicolumn{3}{|c|}{ Univariate } & \multicolumn{3}{|c|}{ Multivariate } \\
\hline & HR & $95 \% \mathrm{CI}$ & p-Value & HR & $95 \%$ CI & p-Value \\
\hline PNI & 0.85 & $0.81-0.89$ & $<001$ & 0.87 & $0.81-0.94$ & $<001$ \\
\hline Age (years) & 1.00 & $0.97-1.02$ & 0.916 & & & \\
\hline Sex (male) & 1.00 & $0.97-1.02$ & 0.916 & & & \\
\hline BMI & 0.89 & $0.81-0.97$ & 0.011 & 0.96 & $0.87-1.06$ & 0.422 \\
\hline $\mathrm{DM}$ & 1.19 & $0.43-3.28$ & 0.738 & & & \\
\hline HTN & 0.70 & $0.22-2.22$ & 0.547 & & & \\
\hline MELD scores & 1.19 & $0.98-1.45$ & 0.088 & 0.94 & $0.74-1.20$ & 0.635 \\
\hline CTP scores & 2.45 & $1.70-3.52$ & $<001$ & 0.97 & $0.49-1.94$ & 0.936 \\
\hline TNM staging & & & $<001$ & & & $<001$ \\
\hline 1 & $\begin{array}{c}1.00 \\
\text { (Ref.) }\end{array}$ & & & $\begin{array}{c}1.00 \\
\text { (Ref.) }\end{array}$ & & \\
\hline 2 & 2.35 & $\begin{array}{l}0.52- \\
10.65\end{array}$ & 0.270 & 2.36 & $\begin{array}{l}0.52- \\
10.79\end{array}$ & 0.270 \\
\hline 3 & 3.92 & $\begin{array}{l}1.23- \\
12.55\end{array}$ & 0.022 & 3.67 & $\begin{array}{l}1.14- \\
11.81\end{array}$ & 0.030 \\
\hline $4 \mathrm{~A}$ & 10.37 & $\begin{array}{l}5.13- \\
20.96\end{array}$ & $<001$ & 8.85 & $\begin{array}{l}4.29- \\
18.22\end{array}$ & $<001$ \\
\hline $4 \mathrm{~B}$ & 19.14 & $\begin{array}{l}7.58- \\
48.30\end{array}$ & $<001$ & 9.26 & $\begin{array}{l}3.42- \\
25.11\end{array}$ & $<001$ \\
\hline Operation time (min) & 1.00 & $1.00-1.00$ & 0.028 & 1.00 & $1.00-1.00$ & 0.713 \\
\hline Total fluids (mL/kg) & 1.02 & $1.00-1.03$ & $<001$ & 1.00 & $0.99-1.02$ & 0.548 \\
\hline Synthetic colloid use & 0.88 & $0.51-1.54$ & 0.662 & & & \\
\hline Urine output (mL/kg/h) & 1.07 & $0.87-1.31$ & 0.515 & & & \\
\hline RBC transfusion & 1.29 & $1.19-1.41$ & $<001$ & 1.03 & $0.44-2.43$ & 0.948 \\
\hline
\end{tabular}

HR: hazard ratio; CI: confidence interval; PNI: prognostic nutritional index; BMI: body mass index; DM: diabetes mellitus; HTN: hypertension; MELD; model for end-stage liver disease; CTP: Child-Turcotte-Pugh; RBC: red blood cell. Values are expressed as mean \pm standard deviation, median (interquartile range), or $\mathrm{n}$ (proportion).

Table 4. Cox regression analyses of five-year mortality.

\begin{tabular}{|c|c|c|c|c|c|c|}
\hline & \multicolumn{3}{|c|}{ Univariate } & \multicolumn{3}{|c|}{ Multivariate } \\
\hline & HR & $95 \%$ CI & $p$-Value & HR & $95 \%$ CI & $p$-Value \\
\hline PNI & 0.91 & $0.88-0.93$ & $<001$ & 0.93 & $0.90-0.97$ & $<001$ \\
\hline Age (years) & 0.99 & $0.98-1.00$ & 0.196 & & & \\
\hline Sex (male) & 1.00 & $0.69-1.44$ & 0.995 & & & \\
\hline BMI & 0.93 & $0.89-0.98$ & 0.004 & 0.97 & $0.92-1.02$ & 0.238 \\
\hline $\mathrm{DM}$ & 1.20 & $0.71-2.02$ & 0.505 & & & \\
\hline HTN & 0.78 & $0.44-1.40$ & 0.411 & & & \\
\hline MELD scores & 1.22 & $1.10-1.35$ & $<001$ & 1.16 & $1.03-1.30$ & 0.015 \\
\hline CTP scores & 1.80 & $1.42-2.28$ & $<001$ & 0.96 & $0.68-1.35$ & 0.824 \\
\hline TNM staging & & & $<001$ & & & $<001$ \\
\hline 1 & $\begin{array}{c}1.00 \\
\text { (Ref.) }\end{array}$ & & & $\begin{array}{c}1.00 \\
\text { (Ref.) }\end{array}$ & & \\
\hline 2 & 2.02 & $1.14-3.57$ & 0.016 & 1.91 & $1.07-3.40$ & 0.028 \\
\hline 3 & 2.04 & $1.09-3.84$ & 0.027 & 1.87 & $0.99-3.53$ & 0.056 \\
\hline $4 \mathrm{~A}$ & 3.47 & $2.57-4.70$ & $<001$ & 3.73 & $2.72-5.11$ & $<001$ \\
\hline $4 \mathrm{~B}$ & 7.28 & $\begin{array}{l}4.18- \\
12.67\end{array}$ & $<001$ & 3.35 & $1.80-6.22$ & $<001$ \\
\hline Operation time (min) & 1.00 & $1.00-1.01$ & $<001$ & 1.00 & $1.00-1.00$ & 0.387 \\
\hline Total fluids (mL/kg) & 1.02 & $1.01-1.02$ & $<001$ & 1.01 & $1.00-1.02$ & 0.088 \\
\hline Synthetic colloid use & 1.32 & $1.00-1.74$ & 0.048 & 1.48 & $1.08-2.03$ & 0.015 \\
\hline Urine output (mL/kg/h) & 1.15 & $1.04-1.28$ & 0.010 & 1.01 & $0.90-1.14$ & 0.873 \\
\hline RBC transfusion & 3.67 & $2.60-5.20$ & $<001$ & 1.61 & $1.00-2.60$ & 0.053 \\
\hline
\end{tabular}

HR: hazard ratio; CI: confidence interval; PNI: prognostic nutritional index; BMI: body mass index; DM: diabetes mellitus; HTN: hypertension; MELD; model for end-stage liver disease; CTP: Child-Turcotte-Pugh; RBC: red blood cell. Values are expressed as mean \pm standard deviation, median (interquartile range), or $\mathrm{n}$ (proportion). 
The preoperative PNI was significantly associated with the incidence of postoperative RRT (OR: $0.76,95 \%$ CI: 0.60 to $0.98, p=0.032$ ) and overall mortality (HR: $0.87,95 \% \mathrm{CI}$ : 0.79 to $0.97, p=0.010$ ), even after adjusting for other potentially confounding variables (Table 4). However, there were no significant associations between the PNI and PHLF and ICU admission (Table 5).

Table 5. AKI incidence and surgical outcomes adjusted by PNI.

\begin{tabular}{ccccc}
\hline & \multicolumn{2}{c}{ Univariate } & \multicolumn{2}{c}{ Multivariate * } \\
\hline & OR (95\% CI) & $p$-Value & OR (95\% CI) & $p$-Value \\
AKI & $0.93(0.89-0.98)$ & 0.004 & $0.92(0.85-0.99)$ & 0.021 \\
Postoperative RRT & $0.82(0.73-0.93)$ & 0.002 & $0.76(0.60-0.98)$ & 0.032 \\
PHLF & $0.88(0.84-0.91)$ & $<0.001$ & $0.94(0.88-1.00)$ & 0.065 \\
ICU admission & $1.02(0.97-1.06)$ & 0.519 & $1.05(0.99-1.12)$ & 0.120 \\
& HR (95\% CI) & $p$-Value & HR (95\% CI) & $p$-Value \\
One-year mortality & $0.85(0.81-0.89)$ & $<001$ & $0.87(0.81-0.94)$ & $<001$ \\
Five-year mortality & $0.91(0.88-0.93)$ & $<001$ & $0.93(0.90-0.97)$ & $<001$ \\
Overall mortality & $0.91(0.85-0.96)$ & 0.002 & $0.87(0.79-0.97)$ & 0.010 \\
\hline
\end{tabular}

* Adjusted for age, sex, body mass index, diabetes mellitus, hypertension, model for end-stage liver disease score, Child-Turcotte-Pugh score, TNM staging, operation time, total fluids, synthetic colloid use, and red blood cell transfusion. AKI: acute kidney injury; PNI: prognostic nutritional index; OR: odds ratio; HR: hazard ratio; CI: confidence interval; RRT; renal replacement therapy; PHLF: post-hepatectomy liver failure; ICU: intensive care unit. Values are expressed as mean \pm standard deviation, median (interquartile range), or $\mathrm{n}$ (proportion).

Figure 2 shows the Kaplan-Meier curve according to the preoperative PNI cutoff value $<45$. The one-year and five-year mortality was significantly higher in the PNI $<45$ group than in the PNI $\geq 45$ group (log-rank test; $p<0.001$ ).

A

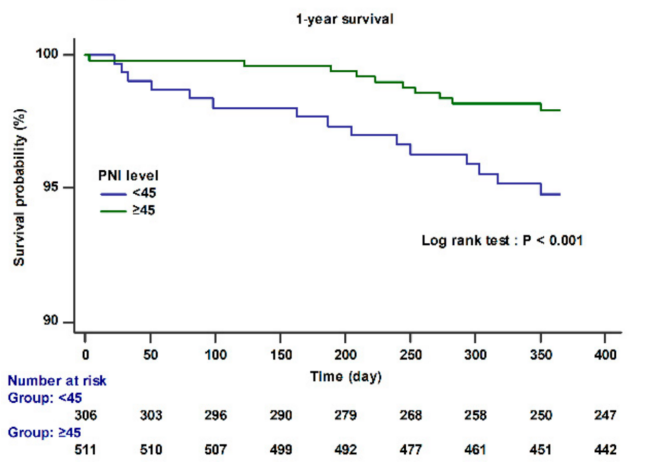

B

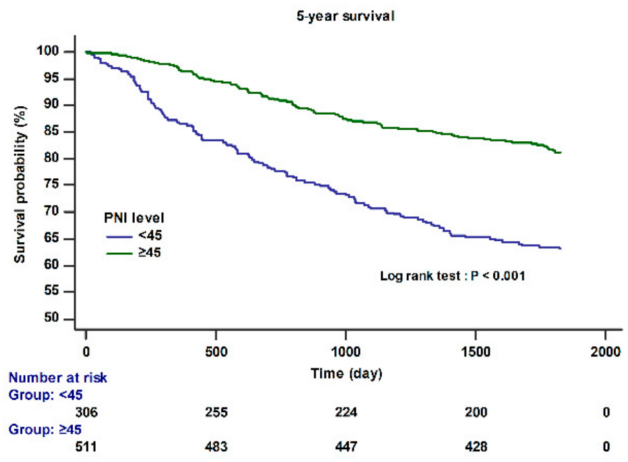

Figure 2. Kaplan-Meier curves for one-year (A) and five-year survival (B) according to the preoperative PNI cutoff value $<45$ (log-rank test; $p<0.001$ ). The proportional hazards assumption was satisfied in Schoenfeld's residual test $(p=0.1828)$.

\section{Discussion}

Our study demonstrated that high preoperative PNI was significantly associated with a lower incidence of postoperative AKI in patients who had undergone open hepatectomy for HCC. In addition, high preoperative PNI was significantly associated with outcomes such as low postoperative RRT requirement, low one-year, five-year and overall mortality. This suggests that the preoperative PNI might be a predictive factor for postoperative AKI and mortality in HCC patients.

Postoperative AKI in HCC patients is closely related to postoperative mortality [9], and a recent study reported postoperative $\mathrm{AKI}$ as the strongest independent predictor of postoperative mortality [8]. Patients with HCC are at high risk of developing postoperative AKI due to the strong association between renal and hepatic dysfunction [2]. In particular, liver cirrhosis is closely related to AKI as it induces a reduction in the effective blood volume due to dilation of the splenic and peripheral blood vessels, leading to systemic 
hypoperfusion and compensatory production of antidiuretic hormones [26,27]. Therefore, many efforts have been made to identify the risk factors for postoperative AKI in HCC patients [9-11]. Slankamenac et al. suggested that blood transfusions, hepaticojejunostomy, and oliguria were the best predictors of AKI after liver surgery [10]. Another study reported preoperative eGFR and hypertension as perioperative risk factors for AKI after liver resection surgery [11]. Lim et al. reported that an increased MELD score, major hepatectomy, and prolonged operation time were risk factors for AKI in patients who underwent hepatectomy for HCC [8]. Recently, biological markers such as the AST/ALT ratio or gamma-glutamyl transferase/alanine aminotransferase ratio (GGT/ALT) have been suggested as predictors of AKI after liver surgery [12,13].

The PNI, an index calculated using the serum albumin level and lymphocyte count, is another biological marker that indicates a patient's nutritional and immune status [15] and is associated with various postoperative complications [28-31]. Min et al. suggested that the modified PNI predicted postoperative AKI within one week in patients receiving living donor liver transplantation, which is better than the predictive ability of the conventional MELD score [32]. To our knowledge, this is the first study to evaluate the association between the preoperative PNI and postoperative AKI in patients with HCC. In this study, a significant association was observed between the PNI and the incidence of postoperative AKI and RRT. These results suggest that the preoperative PNI may be another biological marker that predicts postoperative renal function.

In the multivariate logistic regression analysis, DM and the use of synthetic colloids were also found to be significantly associated with postoperative AKI. DM has been reported as a risk factor for AKI in surgical patients [33,34]. DM is associated with postischemic microangiopathy, interstitial inflammation [34], and conditions that can cause AKI even in the absence of chronic kidney disease [33].

The use of synthetic colloids such as hydroxyethyl starch in the ICU and noncardiac surgery has been associated with AKI [35,36]. The mechanisms of synthetic colloid-induced postoperative AKI are yet to be determined incomplete. However, some experimental models and clinical studies indicate that synthetic colloids can no longer be considered safe [37].

In this study, the preoperative PNI was significantly associated with postoperative AKI; this could be attributed to the unique characteristics of the PNI, a combination of albumin and lymphocyte levels, which reflects both the nutritional status and immune function of the patient. Albumin is reported to have reno-protective properties; it was associated with increased renal perfusion in an animal model [38] and closely associated with proximal tubular homeostasis [39,40] and relief of nephrotoxic effects of drugs in a human study [41]. Lymphocytes are also known to play an important role in the initiation, proliferation, and recovery of AKI [42], and one study showed a significant association between preoperative lymphocytopenia and postoperative AKI in cardiac surgery [43]. Recently, the newly identified renal $\mathrm{T}$ lymphocytes have been reported to have complex functions, such as a potential anti-inflammatory role in AKI [44].

Our study demonstrated that the preoperative PNI was significantly associated with outcomes such as one-year and overall mortality, which is consistent with the results of previous studies on patients who underwent hepatectomy [45-47]. Low serum albumin is common in chronic liver disease and is associated with a low survival rate [48]. A recent randomized clinical trial demonstrated that long-term albumin administration in patients with decompensated cirrhosis improved the overall survival and complications [49]. The lymphocyte count was significantly associated with the overall survival in HCC patients. The lymphocyte/monocyte ratio reflects the immune status of the tumor microenvironment and has been reported to be an independent predictor of survival in HCC patients [50].

There are some limitations to our study. First, our study is retrospective in nature; thus, it is possible that one of the biggest drawbacks of retrospective studies, that is, unconsidered confounding factors, triggered potential biases. However, statistical analysis was performed to minimize the impact of these confounding factors by adjusting for 
variables that could potentially affect the outcomes. Second, our study mostly included data from a single ethnic group within Korea; hence, the results may have been biased due to the involvement of homogeneous groups. Therefore, further research involving heterogeneous groups is needed. Third, to date, there is no exact consensus on the cutoff value of the PNI for postoperative AKI. More well-designed studies on various diseases are required for the accurate validation of preoperative PNI cutoff values that predict postoperative AKI.

\section{Conclusions}

In conclusion, high preoperative PNI was significantly associated with a lower incidence of postoperative AKI and low mortality in patients who had undergone hepatectomy for HCC. These results suggest that the preoperative PNI provides clinically useful predictive information on postoperative AKI and surgical prognosis in HCC patients.

Author Contributions: Conceptualization, formal analysis, methodology, investigation and resources, and writing - original draft, J.H.S.; data curation and validation, I.-G.J.; data curation, methodology, investigation and validation, Y.-J.M.; data curation, and methodology, A.R.J.; data curation, methodology, investigation and validation, S.-H.K.; data curation, and methodology, B.K.; formal analysis, investigation, data curation, visualization, validation, writing - review and editing, supervision, and project administration, J.-G.S. All authors have read and agreed to the published version of the manuscript.

Funding: This research was supported by a grant of the Korea Health Technology R\&D Project through the Korea Health Industry Development Institute (KHIDI), funded by the Ministry of Health \& Welfare, Korea (grant number: HI18C2383).

Institutional Review Board Statement: The study was conducted according to the guidelines of the Declaration of Helsinki, and approved by the Institutional Review Board of Asan Medical Center (protocol no. 2021-0243, date of approval: 15 February 2021).

Informed Consent Statement: The requirement for written informed consent was waived owing to the retrospective nature of this study.

Data Availability Statement: The dataset used and/or analyzed during the current study is available from the corresponding author on reasonable request.

Acknowledgments: We gratefully acknowledge the statistical support of Seonok Kim (Department of Clinical Epidemiology and Biostatistics, Asan Medical Center and University of Ulsan College of Medicine).

Conflicts of Interest: The authors declare no conflict of interest.

\section{References}

1. Peres, L.A.; Bredt, L.C.; Cipriani, R.F. Acute renal injury after partial hepatectomy. World J. Hepatol. 2016, 8, 891-901. [CrossRef] [PubMed]

2. Bressan, A.K.; James, M.T.; Dixon, E.; Bathe, O.F.; Sutherland, F.R.; Ball, C.G. Acute kidney injury following resection of hepatocellular carcinoma: Prognostic value of the acute kidney injury network criteria. Can. J. Surg. 2018, 61, E11-E16. [CrossRef] [PubMed]

3. Slankamenac, K.; Breitenstein, S.; Held, U.; Beck-Schimmer, B.; Puhan, M.A.; Clavien, P.A. Development and validation of a prediction score for postoperative acute renal failure following liver resection. Ann. Surg. 2009, 250, 720-728. [CrossRef] [PubMed]

4. Hobson, C.; Ozrazgat-Baslanti, T.; Kuxhausen, A.; Thottakkara, P.; Efron, P.A.; Moore, F.A.; Moldawer, L.L.; Segal, M.S.; Bihorac, A. Cost and Mortality Associated With Postoperative Acute Kidney Injury. Ann. Surg. 2015, 261, 1207-1214. [CrossRef]

5. Bihorac, A.; Yavas, S.; Subbiah, S.; Hobson, C.E.; Schold, J.D.; Gabrielli, A.; Layon, A.J.; Segal, M.S. Long-term risk of mortality and acute kidney injury during hospitalization after major surgery. Ann. Surg. 2009, 249, 851-858. [CrossRef]

6. Chertow, G.M.; Burdick, E.; Honour, M.; Bonventre, J.V.; Bates, D.W. Acute kidney injury, mortality, length of stay, and costs in hospitalized patients. J. Am. Soc. Nephrol. 2005, 16, 3365-3370. [CrossRef]

7. Palomba, H.; Castro, I.; Yu, L.; Burdmann, E.A. The duration of acute kidney injury after cardiac surgery increases the risk of long-term chronic kidney disease. J. Nephrol. 2017, 30, 567-572. [CrossRef] 
8. Lim, C.; Audureau, E.; Salloum, C.; Levesque, E.; Lahat, E.; Merle, J.C.; Compagnon, P.; Dhonneur, G.; Feray, C.; Azoulay, D. Acute kidney injury following hepatectomy for hepatocellular carcinoma: Incidence, risk factors and prognostic value. HPB 2016, 18, 540-548. [CrossRef]

9. Saner, F. Kidney failure following liver resection. Transplant. Proc. 2008, 40, 1221-1224. [CrossRef]

10. Slankamenac, K.; Beck-Schimmer, B.; Breitenstein, S.; Puhan, M.A.; Clavien, P.A. Novel prediction score including pre- and intraoperative parameters best predicts acute kidney injury after liver surgery. World J. Surg. 2013, 37, 2618-2628. [CrossRef]

11. Tomozawa, A.; Ishikawa, S.; Shiota, N.; Cholvisudhi, P.; Makita, K. Perioperative risk factors for acute kidney injury after liver resection surgery: An historical cohort study. Can. J. Anaesth. 2015, 62, 753-761. [CrossRef]

12. Xu, J.; Xia, Y.; Li, S.; Cheng, X.; Hu, S.; Gao, Y.; Zhou, X.; Wang, G.; Zheng, Q. A retrospective pilot study to examine the potential of aspartate aminotransferase to alanine aminotransferase ratio as a predictor of postoperative acute kidney injury in patients with hepatocellular carcinoma. Ann. Clin. Biochem. 2019, 56, 357-366. [CrossRef] [PubMed]

13. Zhou, X.; Wang, L.; Wang, G.; Cheng, X.; Hu, S.; Ke, W.; Li, M.; Zhang, Y.; Song, Z.; Zheng, Q. A new plasma biomarker enhance the clinical prediction of postoperative acute kidney injury in patients with hepatocellular carcinoma. Clin. Chim. Acta 2017, 475, 128-136. [CrossRef]

14. Onodera, T.; Goseki, N.; Kosaki, G. Prognostic nutritional index in gastrointestinal surgery of malnourished cancer patients. Nihon Geka Gakkai Zasshi 1984, 85, 1001-1005.

15. Cheng, Y.L.; Sung, S.H.; Cheng, H.M.; Hsu, P.F.; Guo, C.Y.; Yu, W.C.; Chen, C.H. Prognostic Nutritional Index and the Risk of Mortality in Patients With Acute Heart Failure. J. Am. Heart Assoc. 2017, 6. [CrossRef]

16. Hu, Y.; Cao, Q.; Wang, H.; Yang, Y.; Xiong, Y.; Li, X.; Zhou, Q. Prognostic nutritional index predicts acute kidney injury and mortality of patients in the coronary care unit. Exp. Ther. Med. 2021, 21, 123. [CrossRef]

17. Salati, M.; Filippi, R.; Vivaldi, C.; Caputo, F.; Leone, F.; Salani, F.; Cerma, K.; Aglietta, M.; Fornaro, L.; Sperti, E.; et al. The prognostic nutritional index predicts survival and response to first-line chemotherapy in advanced biliary cancer. Liver Int. 2020, 40, 704-711. [CrossRef]

18. Zencirkiran Agus, H.; Kahraman, S. Prognostic nutritional index predicts one-year outcome in heart failure with preserved ejection fraction. Acta Cardiol. 2020, 75, 450-455. [CrossRef]

19. Pinato, D.J.; North, B.V.; Sharma, R. A novel, externally validated inflammation-based prognostic algorithm in hepatocellular carcinoma: The prognostic nutritional index (PNI). Br. J. Cancer 2012, 106, 1439-1445. [CrossRef] [PubMed]

20. Wang, D.; Hu, X.; Xiao, L.; Long, G.; Yao, L.; Wang, Z.; Zhou, L. Prognostic Nutritional Index and Systemic Immune-Inflammation Index Predict the Prognosis of Patients with HCC. J. Gastrointest. Surg. 2021, 25, 421-427. [CrossRef] [PubMed]

21. Germain, T.; Favelier, S.; Cercueil, J.P.; Denys, A.; Krausé, D.; Guiu, B. Liver segmentation: Practical tips. Diagn. Interv. Imaging 2014, 95, 1003-1016. [CrossRef]

22. Vibert, E.; Perniceni, T.; Levard, H.; Denet, C.; Shahri, N.K.; Gayet, B. Laparoscopic liver resection. Br. J. Surg. 2006, 93, 67-72. [CrossRef]

23. Hwang, D.W.; Han, H.S.; Yoon, Y.S.; Cho, J.Y.; Kwon, Y.; Kim, J.H.; Park, J.S.; Yoon, D.S.; Choi, I.S.; Ahn, K.S.; et al. Laparoscopic major liver resection in Korea: A multicenter study. J. Hepatobiliary Pancreat. Sci. 2013, 20, 125-130. [CrossRef]

24. Section 2: AKI Definition. Kidney Int. Suppl. 2012, 2, 19-36. [CrossRef]

25. Rahbari, N.N.; Garden, O.J.; Padbury, R.; Brooke-Smith, M.; Crawford, M.; Adam, R.; Koch, M.; Makuuchi, M.; Dematteo, R.P.; Christophi, C.; et al. Posthepatectomy liver failure: A definition and grading by the International Study Group of Liver Surgery (ISGLS). Surgery 2011, 149, 713-724. [CrossRef]

26. Henriksen, J.H.; Bendtsen, F.; Gerbes, A.L.; Christensen, N.J.; Ring-Larsen, H.; Sørensen, T.I. Estimated central blood volume in cirrhosis: Relationship to sympathetic nervous activity, beta-adrenergic blockade and atrial natriuretic factor. Hepatology 1992, 16, 1163-1170. [CrossRef]

27. Henriksen, J.H.; Bendtsen, F.; Sørensen, T.I.; Stadeager, C.; Ring-Larsen, H. Reduced central blood volume in cirrhosis. Gastroenterology 1989, 97, 1506-1513. [CrossRef]

28. Kanda, M.; Fujii, T.; Kodera, Y.; Nagai, S.; Takeda, S.; Nakao, A. Nutritional predictors of postoperative outcome in pancreatic cancer. Br. J. Surg. 2011, 98, 268-274. [CrossRef]

29. Mohri, Y.; Inoue, Y.; Tanaka, K.; Hiro, J.; Uchida, K.; Kusunoki, M. Prognostic nutritional index predicts postoperative outcome in colorectal cancer. World J. Surg. 2013, 37, 2688-2692. [CrossRef]

30. Nakatani, M.; Migita, K.; Matsumoto, S.; Wakatsuki, K.; Ito, M.; Nakade, H.; Kunishige, T.; Kitano, M.; Kanehiro, H. Prognostic significance of the prognostic nutritional index in esophageal cancer patients undergoing neoadjuvant chemotherapy. Dis. Esophagus. 2017, 30, 1-7. [CrossRef]

31. Yu, J.; Hong, B.; Park, J.Y.; Hwang, J.H.; Kim, Y.K. Impact of Prognostic Nutritional Index on Postoperative Pulmonary Complications in Radical Cystectomy: A Propensity Score-Matched Analysis. Ann. Surg. Oncol. 2021, 28, 1859-1869. [CrossRef] [PubMed]

32. Min, J.Y.; Woo, A.; Chae, M.S.; Hong, S.H.; Park, C.S.; Choi, J.H.; Chung, H.S. Predictive Impact of Modified-Prognostic Nutritional Index for Acute Kidney Injury within 1-week after Living Donor Liver Transplantation. Int. J. Med. Sci. 2020, 17, 82-88. [CrossRef] [PubMed]

33. James, M.T.; Grams, M.E.; Woodward, M.; Elley, C.R.; Green, J.A.; Wheeler, D.C.; de Jong, P.; Gansevoort, R.T.; Levey, A.S.; Warnock, D.G.; et al. A Meta-analysis of the Association of Estimated GFR, Albuminuria, Diabetes Mellitus, and Hypertension With Acute Kidney Injury. Am. J. Kidney Dis. 2015, 66, 602-612. [CrossRef] [PubMed] 
34. Patschan, D.; Müller, G.A. Acute Kidney Injury in Diabetes Mellitus. Int. J. Nephrol. 2016, 2016, 6232909. [CrossRef]

35. Kashy, B.K.; Podolyak, A.; Makarova, N.; Dalton, J.E.; Sessler, D.I.; Kurz, A. Effect of hydroxyethyl starch on postoperative kidney function in patients having noncardiac surgery. Anesthesiology 2014, 121, 730-739. [CrossRef]

36. Myburgh, J.A.; Finfer, S.; Bellomo, R.; Billot, L.; Cass, A.; Gattas, D.; Glass, P.; Lipman, J.; Liu, B.; McArthur, C.; et al. Hydroxyethyl starch or saline for fluid resuscitation in intensive care. N. Engl. J. Med. 2012, 367, 1901-1911. [CrossRef]

37. Schortgen, F.; Brochard, L. Colloid-induced kidney injury: Experimental evidence may help to understand mechanisms. Crit. Care 2009, 13, 130. [CrossRef]

38. Kaufmann, M.A.; Castelli, I.; Pargger, H.; Drop, L.J. Nitric oxide dose-response study in the isolated perfused rat kidney after inhibition of endothelium-derived relaxing factor synthesis: The role of serum albumin. J. Pharmacol. Exp. Ther. 1995, $273,855-862$.

39. Lee, E.H.; Baek, S.H.; Chin, J.H.; Choi, D.K.; Son, H.J.; Kim, W.J.; Hahm, K.D.; Sim, J.Y.; Choi, I.C. Preoperative hypoalbuminemia is a major risk factor for acute kidney injury following off-pump coronary artery bypass surgery. Intensive Care Med. 2012, 38, 1478-1486. [CrossRef]

40. Li, N.; Qiao, H.; Guo, J.F.; Yang, H.Y.; Li, X.Y.; Li, S.L.; Wang, D.X.; Yang, L. Preoperative hypoalbuminemia was associated with acute kidney injury in high-risk patients following non-cardiac surgery: A retrospective cohort study. BMC Anesthesiol. 2019, 19, 171. [CrossRef]

41. Gamba, G.; Contreras, A.M.; Cortés, J.; Nares, F.; Santiago, Y.; Espinosa, A.; Bobadilla, J.; Jiménez Sánchez, G.; López, G.; Valadez, A.; et al. Hypoalbuminemia as a risk factor for amikacin nephrotoxicity. Rev. Investig. Clin. 1990, 42, $204-209$.

42. Weller, S.; Varrier, M.; Ostermann, M. Lymphocyte Function in Human Acute Kidney Injury. Nephron 2017, 137, 287-293. [CrossRef]

43. Aghdaii, N.; Ferasatkish, R.; Mohammadzadeh Jouryabi, A.; Hamidi, S.H. Significance of preoperative total lymphocyte count as a prognostic criterion in adult cardiac surgery. Anesth. Pain Med. 2014, 4, e20331. [CrossRef]

44. Martina, M.N.; Noel, S.; Bandapalle, S.; Hamad, A.R.; Rabb, H. T lymphocytes and acute kidney injury: Update. Nephron Clin. Pract. 2014, 127, 51-55. [CrossRef]

45. Fan, X.; Chen, G.; Li, Y.; Shi, Z.; He, L.; Zhou, D.; Lin, H. The Preoperative Prognostic Nutritional Index in Hepatocellular Carcinoma After Curative Hepatectomy: A Retrospective Cohort Study and Meta-Analysis. J. Investig. Surg. 2019, 1-8. [CrossRef]

46. Imai, D.; Maeda, T.; Shimokawa, M.; Wang, H.; Yoshiya, S.; Takeishi, K.; Itoh, S.; Harada, N.; Ikegami, T.; Yoshizumi, T.; et al. Prognostic nutritional index is superior as a predictor of prognosis among various inflammation-based prognostic scores in patients with hepatocellular carcinoma after curative resection. Hepatol. Res. 2020, 50, 101-109. [CrossRef]

47. Okamura, Y.; Ashida, R.; Ito, T.; Sugiura, T.; Mori, K.; Uesaka, K. Preoperative neutrophil to lymphocyte ratio and prognostic nutritional index predict overall survival after hepatectomy for hepatocellular carcinoma. World J. Surg. 2015, 39, 1501-1509. [CrossRef]

48. Salerno, F.; Borroni, G.; Moser, P.; Badalamenti, S.; Cassarà, L.; Maggi, A.; Fusini, M.; Cesana, B. Survival and prognostic factors of cirrhotic patients with ascites: A study of 134 outpatients. Am. J. Gastroenterol. 1993, 88, 514-519.

49. Caraceni, P.; Riggio, O.; Angeli, P.; Alessandria, C.; Neri, S.; Foschi, F.G.; Levantesi, F.; Airoldi, A.; Boccia, S.; Svegliati-Baroni, G.; et al. Long-term albumin administration in decompensated cirrhosis (ANSWER): An open-label randomised trial. Lancet 2018, 391, 2417-2429. [CrossRef]

50. Mano, Y.; Yoshizumi, T.; Yugawa, K.; Ohira, M.; Motomura, T.; Toshima, T.; Itoh, S.; Harada, N.; Ikegami, T.; Soejima, Y.; et al. Lymphocyte-to-Monocyte Ratio Is a Predictor of Survival After Liver Transplantation for Hepatocellular Carcinoma. Liver Transpl. 2018, 24, 1603-1611. [CrossRef] 